REVIEW

\title{
Dasatinib in chronic myeloid leukemia: a review
}

\author{
Dolly G Aguilera' \\ Apostolia MTsimberidou ${ }^{2}$ \\ 'Department of Hematology- \\ Oncology and Stem Cell \\ Transplantation, Children's Memorial \\ Hospital, Northwestern University, \\ Chicago, IL, USA; ${ }^{2}$ Department of \\ Investigational Cancer Therapeutics, \\ The University of Texas MD \\ Anderson Cancer Center, Houston \\ Texas USA
}

Correspondence: Apostolia MTsimberidou Assistant Professor, Department of Investigational Cancer Therapeutics, The University of Texas MD Anderson Cancer Center, 1515 Holcombe Boulevard, Unit 455, Houston,

Texas 77030, USA

Tel +l 7|37924259

Email atsimber@mdanderson.org
Abstract: Deregulated BCR-ABL tyrosine kinase (TK) activity is the molecular marker for chronic myeloid leukemia (CML), which provides an identifiable target for developing therapeutic agents. Imatinib mesylate, a BCR-ABL TK inhibitor, is the frontline therapy for CML. Despite the stunning efficacy of this agent, a small number of patients develop a suboptimal response or resistance to imatinib. In newly diagnosed patients with chronic phase CML, the rate of resistance to imatinib at 4 years was up to $20 \%$, increasing to $70 \%$ to $90 \%$ for patients in the accelerated/blastic phase. Resistance to imatinib led to the development of novel TK inhibitors such as dasatinib. Several clinical trials have reported more durable complete hematologic and cytogenetic responses with this agent in patients who are resistant or intolerant to imatinib. Dasatinib is well tolerated and has broad efficacy, resulting in durable responses in patients with any BCR-ABL mutation except for T3151 and mutations in codon 317 - most commonly F317L - including mutations that were highly resistant to imatinib, such as L248, Y253, E255, F359, and H396. Dasatinib is recommended for CML in chronic, blastic or accelerated phase that is resistant or intolerant to imatinib. Dasatinib was approved by the FDA at $100 \mathrm{mg}$ once daily as the starting dose in patients with chronic phase CML and at $70 \mathrm{mg}$ twice daily in patients with accelerated or blastic phase CML. Various clinical trial results provided evidence that resistance to one TK inhibitor can be reversed with the use of a different TK inhibitor (TKI). Other second-generation TKIs with activity in CML include nilotinib, bosutinib and INNO 406. New molecules, such as the inhibitor of Aurora family serine-threonine kinases, MK0457, which has antileukemic activity in CML associated with a T315I mutation, are being investigated. Allogeneic hematopoietic stem cell transplantation remains an option for selected patients.

Keywords: dasatininb, chronic myeloid leukemia, BCR-ABL, tyrosine kinase inhibitor

\section{Introduction}

Chronic myeloid leukemia (CML) accounts for $20 \%$ of all adult leukemias, approximately 4300 cases per year in the United States, with a median patient age of 45 to 55 years. Most patients are diagnosed in the chronic phase and can progress to the accelerated or blastic phase, which is associated with decreased survival. ${ }^{1}$ The characteristic cytogenetic abnormality underlying CML is the Philadelphia chromosome (Ph), which corresponds to the reciprocal translocation between chromosomes 9 and 22. This translocation causes the Abelson $t k$ gene $(A b l)$ to fuse with the breakpoint cluster region of the $B c r$ gene.

The $B C R-A B L$ oncogene triggers intracellular signaling, activating multiple transduction cascades, promoting the growth, proliferation and survival of hematopoietic cells. ${ }^{2}$ BCR-ABL plays a role in defective DNA repair, alteration of cellular adhesion and inhibition of apoptosis. ${ }^{3}$

Inhibiting BCR-ABL tyrosine kinase activity leads to the induction of apoptosis and inhibits cellular proliferation in vitro. ${ }^{4}$ Degregulated BCR-ABL tyrosine kinase activity is the molecular marker for CML, which provides an identifiable target for developing therapeutic agents. Various BCR-ABL isoforms with diverse molecular weights have been identified. The weights range from 185 to $230 \mathrm{kDa}$ depending on the translocation breakpoints in the $B c r$ gene and the mRNA splicing site. ${ }^{5}$ The $210 \mathrm{kDa}$ BCR-ABL protein is the most common Bcr breakpoint in CML. 


\section{Imatinib mesylate}

Imatinib mesylate (Gleevec ${ }^{\circledR}$; Novartis Pharma, East Hanover, NJ, USA) is a very specific inhibitor of BCR-ABL tyrosine kinase activity of the 2-phenylamino pyrimidine class, and it is approved by the Food and Drug Administration as frontline therapy for patients with CML. ${ }^{6}$ Imatinib acts by binding and stabilizing the inactive form of BCR-ABL, thereby inhibiting its autophosphorylation and the phosphorylation of its substrate, abrogating proliferation and inducing apoptosis of BCR-ABL-positive cells., ${ }^{4,7}$ The superiority of imatinib for successful clinical outcomes of patients with CML was confirmed by the International Randomized Study of Interferon plus low-dose cytarabine, the previous standard of care, vs STI571, or the imatinib (IRIS) trial, in which 553 patients were randomized to each arm. ${ }^{8}$ Imatinib induced high rates of complete hematologic response (96\% at 1 year, which increased to $98 \%$ at 5 years), ${ }^{8}$ a major cytogenetic response ( $86 \%$ at 1 year, which increased to $92 \%$ at 5 years), a complete cytogenetic response (69\% at 1 year and $87 \%$ at 5 years), a progression-free survival rate without accelerated or blast crisis ( $93 \%$ at 6 years), overall progression-free survival ( $83 \%$ at 6 years) and overall survival ( $95 \%$ at 6 years, taking into consideration only CML-related deaths; and $88 \%$ at 6 years when deaths from any cause were included). ${ }^{8-10}$

However, a small number of patients develop a suboptimal response or resistance to imatinib. In newly diagnosed patients with chronic phase CML, the rate of resistance to imatinib at 4 years was $20 \%$, increasing to $70 \%$ to $90 \%$ for patients in the accelerated/blastic phase. ${ }^{8}$ Criteria for failure to respond to imatinib have been defined as no hematologic response at 3 months, incomplete hematologic response or no cytogenetic response at 6 months, less than a partial cytogenetic response at 12 months, less than a complete cytogenetic response at 18 months, and in the case of a hematologic or cytogenetic response, loss, or appearance of highly imatinibresistant $B C R-A B L$ mutations. A suboptimal response was defined as an incomplete hematologic response at 3 months, less than a partial cytogenetic response at 6 months, less than a complete cytogenetic response at 12 months, less than a major molecular response at 18 months, and, in the case of a major molecular response, loss of BCR-ABL, other mutations or other chromosomal abnormalities. ${ }^{11}$

Resistance to imatinib is primary (no response after initial treatment) or secondary (after an objective response has been achieved). The latter has been attributed to point mutations in the kinase domain of BCR-ABL consisting of amino-acid substitutions that impair imatinib binding but retain kinase activity. ${ }^{12}$ The T315I mutation in the ATP binding pocket of the ABL tyrosine kinase with its single amino acid substitution of the threonine 315 residue with isoleucine was initially reported to cause resistance to imatinib and later to dasatinib. ${ }^{13}$ However, in 2005 , more than 30 point mutations composed of single amino acid substitutions in the BCR-ABL kinase domain were identified in patients with relapsed disease. Additionally, the proportion of mutations in the late chronic phase of CML and accelerated or blastic phases range from $22 \%$ to $53 \%$, whereas such mutations occur infrequently in the early chronic phase. ${ }^{14} \mathrm{~A}$ decrease in sensitivity to imatinib from threefold to greater than 100-fold has been seen after kinase domain mutations reactivate the kinase activity of the BCR-ABL fusion protein.

Other mechanisms of resistance to imatinib include alteration of the balance favoring the active state of $\mathrm{ABL}$ kinase, shifting the equilibrium between active and inactive states $;^{15}$ a change in the conformation of the BCR-ABL protein, preventing imatinib from directly binding to its binding site; ${ }^{16}$ amplification of the $B C R-A B L$ gene, leading to over-expression of BCR-ABL protein, as detected by FISH; ${ }^{13,16}$ or over-expression of the P-glycoprotein efflux pump, for which imatinib is the substrate. ${ }^{17,18}$ Additional mechanisms include the binding of the A1 acid glycoprotein (AGP) to imatinib in plasma, reducing its intracellular concentrations,${ }^{19}$ or a less understood mechanism, the inhibition of ABCG2 protein functionality by imatinib. ${ }^{20}$

Independent $B C R-A B L$ cytogenetic mutations can also contribute to imatinib resistance, including aneuploidy, reciprocal translocations, and $17 \mathrm{p}$ mutations, which lead to the loss of one p53 allele. ${ }^{16}$ Dasatinib was one of the newer tyrosine kinase inhibitors (TKIs) whose development was geared toward overcoming imatinib resistance.

\section{Dasatinib}

Dasatinib (BMS-354825, Sprycel ${ }^{\circledR}$; Bristol Myers Squibb, New York, NY, USA) is a potent, second-generation, small-molecule, multitarget kinase inhibitor of BCR-ABL. This agent exerts greater in vitro activity against unmutated ABL kinase than imatinib. Dasatinib was initially developed as an inhibitor of the Src family of kinases such as Fyn, Yes, Src, and Lyk, but it also inhibits BCR-ABL, EphA2, plateletderived growth factor receptor, and c-Kit. Additionally, it binds to other tyrosine and serine/threonine kinases such as the mitogen-activated protein kinases and the receptor tyrosine kinase, discoidin domain receptor $1 .{ }^{21}$ Dasatinib can inhibit the proliferation and kinase activity of wild-type and BCR-ABL mutant cell lines that are resistant to imatinib, except those carrying the T315I mutation. ${ }^{22,23}$ In vivo studies 
have shown that dasatinib is 325 -fold more potent than imatinib and 16-fold more potent than nilotinib, another BCR-ABL kinase inhibitor, against unmutated BCR-ABL. ${ }^{24}$ In murine models dasatinib was shown to inhibit leukemic cell growth and to prolong the survival of mice harboring wild-type $B C R-A B L$ and M351T, but not the T315I mutant. ${ }^{22}$ Dasatinib is a dual-specific Src and Abl inhibitor that can inhibit both the active and inactive conformations of ABL. ${ }^{22}$ Recently, the activity of dasatinib against 18 imatinib-resistant BCR/ABL mutants in comparison with two other TKIs (nilotinib and bosutinib) was investigated. ${ }^{25}$ Dasatinib was shown to compare favorably to nilotinib against 252 and 253 mutants, which are common in imatinib-resistant patients. ${ }^{26,27}$ Also, it was active against the H396P/R mutation in the activation loop or F359F in the active site. In contrast, it was less active than bosutinib for Q252H and L384M. ${ }^{25}$ In June 2006, the Food and Drug Administration approved dasatinib for the treatment of chronic phase, accelerated phase, or blastic phase CML, resistant or intolerant to imatinib, and for $\mathrm{Ph}$ chromosome-positive acute lymphoid leukemia that was resistant or intolerant to prior therapy. ${ }^{28}$

\section{Mechanism of action}

Dasatinib binds to the ATP-binding site, but extends in the opposite direction from imatinib. Dasatinib binds the inactive and active conformation of the ABL kinase domain, requires fewer contact points with $\mathrm{ABL}$, and has a greater affinity to the ABL kinase domain compared to imatinib. In vitro, dasatinib has demonstrated greater than 325-fold activity against $B C R$ - $A B L$ wild-type, ${ }^{23}$ and the kinase activity of 14 of 15 BCR-ABL imatinib-resistant isoforms has been successfully inhibited at nanomolar concentrations of dasatinib. $^{22,23}$ The activity of dasatinib against several $A B L$ kinase mutations is explained by the fact that it does not require interaction with some of the residues involved in those mutations. Dasatinib's inhibiting potential against Src family kinase members is greater $\left(\mathrm{IC}_{50} 0.5 \mathrm{nmol} / \mathrm{L}\right)$ than its inhibitory activity against $\mathrm{ABL}(1 \mathrm{nmol} / \mathrm{L}) .{ }^{29}$ At nanomolar concentrations, dasatinib also inhibits c-KIT, platelet-derived growth factor receptor and EphA2. Potent Src inhibitory activity of dasatinib in CML progenitors involves BCRABL-dependent and BCR-ABL-independent Src activity. ${ }^{30}$ Dasatinib also inhibits downstream signaling pathways in CML progenitors, ${ }^{30}$ as well as P-mitogen-activated protein kinase (MAPK), P-Akt, and P-STAT5 levels in CML progenitors in the absence but not in the presence of growth factors. In addition, dasatinib significantly suppressed CML colonyforming cells and long-term culture-initiating cells. ${ }^{30}$
Recently, dasatinib-induced BCR-ABL inhibition was shown to be sufficient to commit chronic myeloid leukemia cells irreversibly to apoptosis and suggested that in patients with CML receiving dasatinib once daily, response correlates with the magnitude of BCR-ABL kinase inhibition, demonstrating the potential clinical utility of intermittent potent kinase inhibitor therapy. ${ }^{31}$

Dasatinib was also shown to significantly suppress CML colony-forming cells and long-term culture-initiating cells, but it did not significantly alter the level of apoptosis-regulating proteins in CML CD34 + cells. ${ }^{30}$ Therefore, dasatinib suppresses progenitor growth through inhibition of proliferation and a modest increase in apoptosis in dividing progenitors. ${ }^{30}$ In addition, dasatinib did not enhance suppression and targeting of CML primitive and committed progenitors. ${ }^{30}$

Dasatinib was shown to significantly inhibit CrKL phosphorylation and to be more effective than imatinib mesylate by targeting an earlier progenitor population than imatinib, but without affecting the most primitive quiescent CML cells, which appeared to be resistant to both drugs. ${ }^{32}$

An intriguing observation is that dasatinib may modulate or suppress T-cell activation and proliferation. ${ }^{24}$ In a series of 18 patients treated with dasatinib, 8 patients developed chronic peripheral lymphocytosis, evidenced by natural killer (NK) cells or natural killer/T [NK/T]-cells with large granular lymphocyte morphologies and expression of CD16(+), CD56(+), CD3(-) or CD3(+). More importantly, all patients who developed large granular lymphocyte lymphocytosis achieved optimal molecular responses (8/8 in large granular lymphocytes $(+)$ cases vs 3/10 in large granular lymphocytes $(-)$ cases, $p=0.002$ ). In addition, NK cell cytotoxicity in dasatinib responders was superior to that in non-responders. These findings may have implications for treatment, as large granular lymphocytes might have a therapeutic effect on $\mathrm{Ph}(+)$ leukemic cells. ${ }^{24}$

Potential mechanisms for the pathogenesis of large granular lymphocytes after dasatinib therapy include the reduction of $\mathrm{BCR} / \mathrm{ABL}$ transcription by TKIs and restoration of NK cell numbers and/or functions, and direct activation or modulation of the proliferation and function of LGLs. ${ }^{33}$ However, it is still unclear whether the effect of dasatinib on $\mathrm{NK}$ or NK/T cell proliferation or activation is mediated via Src kinase or other unknown potential pathways involved in the proliferation and differentiation of $\mathrm{NK}$ or $\mathrm{NK} / \mathrm{T}$ cells. ${ }^{33}$

\section{Pharmacokinetics}

Dasatinib is administered orally and peaks from 0.5 to 6 hours after oral dosing. Its half-life is 3 to 5 hours and the absorption 
is not affected by food intake. Dasatinib is metabolized by the cytochrome P450 (CYP) 3A4 isozyme into active and inactive metabolites. Therefore, concomitant use of dasatinib with inducers of CYP3A4 can decrease dasatinib exposure, whereas inhibitors of the 3A4 enzyme, such as antiretrovirals, azole antifungals and macrolides, can increase the toxicity of dasatinib. Recent data suggest that dasatinib given orally crosses the blood-brain barrier. The concentrations of dasatinib found in the cerebrospinal fluid of patients ranged from 1.4 to $20.1 \mathrm{nM}$, and were consistent with the observed antitumor activity in the central nervous system. ${ }^{34}$

Dasatinib was shown to have a high intrinsic permeability in Caco-2 cells, ie, cells derived from a human colon carcinoma, and their growth forms a confluent monolayer on a semipermeable membrane, polarized to resemble the intestinal epithelium. ${ }^{35}$ Although the efflux ratio indicated that dasatinib might be a substrate for an intestinal efflux transporter, in vivo studies in P-gp knockout mice vs wildtype mice showed no difference in the amount of dasatinib remaining unabsorbed in the gastrointestinal tract, suggesting that P-gp may not be responsible for the incomplete bioavailability. ${ }^{35}$

In addition, although the ability of the $\mathrm{ABC}$ transporter protein ABCG2 to transport TKIs is controversial, some investigators have shown that TKIs can inhibit transporter molecules on primary stem cells. ${ }^{36}$

The elimination of dasatinib is primarily via the feces. The concomitant use of $\mathrm{H} 2$ blockers and proton pump inhibitors is not recommended since the solubility of dasatinib depends on intestinal $\mathrm{pH}$, and therefore, anti-acid agents reduce the concentration of dasatinib by approximately $60 \%$. No clinically relevant data are available for dose modifications based on age, gender or ethnicity, although patients greater than 65 years showed increased fluid retention over younger patients. More importantly, the efficacy of dasatinib appears to be independent of patient age.

\section{Clinical trials}

\section{Phase I trials}

In a phase I study dasatinib was administered in 84 patients with $\mathrm{CML}$ or $\mathrm{Ph}(+) \mathrm{ALL}$ who were resistant or intolerant to imatinib, at doses ranging from 15 to $240 \mathrm{mg} /$ day, orally once or twice daily over a 4-week treatment cycle (Table 1). The response rate was dependent on the CML phase. In chronic phase CML, the rates of complete major hematologic response and major cytogenetic response were $92 \%$ and $45 \%$, respectively. The respective rates in patients with accelerated phase CML were $82 \%$ and $27 \%$. Eighty percent of CML patients with lymphoid blastic crisis or Ph(+) ALL had a major hematologic response and a major cytogenetic response. Overall, 95\% of patients in the chronic phase had a durable response compared with $82 \%$ of patients in the accelerated phase. However, within 6 months of therapy, almost all patients with $\mathrm{Ph}(+)$ ALL had relapsed. All $B C R-A B L$ genotypes, with the exception of those with the T315I mutation, responded to dasatinib. The most common toxicity, although not dose-limiting, was reversible myelosuppression. The maximum tolerated dose was not determined; however, most of the complete hematologic responses were noted at daily doses of dasatinib of $\geq 50 \mathrm{mg}$, whereas most cytogenetic responses were noted at higher doses. ${ }^{37}$ The encouraging results of this phase I study led to studies comparing the efficacy of dasatinib with imatinib and other trials.

\section{Phase II trials}

The effects of dasatinib in different stages of CML and $\mathrm{Ph}(+)$ ALL were explored in the START (Src/ABL Tyrosine Kinase Inhibition Activity Research Trials of dasatinib) Program, which included 5 arms: START-A (accelerated phase), START-B (myeloid blast crisis), START-C (chronic phase), START-L (lymphoid blast crisis), and START-R (dasatinib vs high-dose imatinib) (Table 1). Preliminary results of 4 of the 5 single arm phase II studies (A, B, C and $\mathrm{L}$ ) constituted the basis for the new drug application for the indication of dasatinib in CML. These four singlearm, multicenter studies led to the approval of dasatinib by the FDA and included a total of 445 patients with CML in accelerated phase, myeloid phase, and lymphoid blast phase, and $\mathrm{Ph}(+) \mathrm{ALL}$, who were resistant or intolerant to prior therapy, including imatinib. ${ }^{28}$ Dasatinib was administered at an initial dose of $70 \mathrm{mg}$ po twice daily and escalation to $100 \mathrm{mg}$ po twice daily was permitted in the absence of a significant toxicity. Escalation was recommended for increasing leukocyte counts, lack of complete hematologic remission within 1 month from initiation of therapy, lack of complete cytogenetic response after 3 months of therapy, and/ or loss of response. In chronic phase CML (endpoint, major cytogenetic response), the major cytogenetic response rate was $45 \%$ with a complete cytogenetic response rate of $33 \%$; and in accelerated phase CML, myeloid CML, lymphoid blast $\mathrm{CML}$, and $\mathrm{Ph}(+)$ ALL (endpoint, major hematologic response), the rates of major hematologic response were $59 \%, 32 \%, 31 \%$ and $42 \%$, respectively. ${ }^{28}$ As expected, the response rates were higher in patients who were intolerant to imatinib than in those who were imatinib-resistant. ${ }^{28}$ 


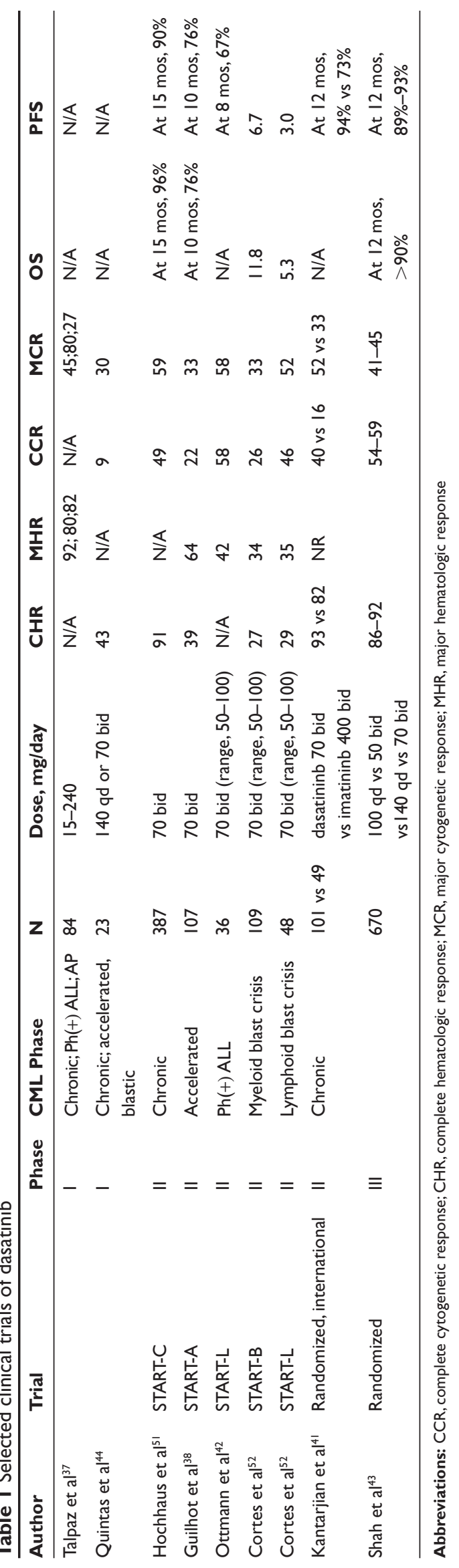

In the open label, phase II (START-A) study of patients with accelerated phase CML who were resistant or intolerant to imatinib, dasatinib $70 \mathrm{mg}$ po twice daily was administered in 107 patients. ${ }^{38}$ At 8 months, the rate of hematologic response was $81 \%$ (major $64 \%$, complete $39 \%$ ). The rates of major and complete cytogenetic response were $33 \%$ and $24 \%$, respectively. At 8 months, of 69 patients who achieved a major cytogenetic response, 7 progressed and the estimated rates of survival and progression-free survival were $76 \%$ each. Severe neutropenia was noted in $76 \%$ of patients, thrombocytopenia in $82 \%$, and anemia in $69 \%$. Cytopenias were reversible with dose reduction or interruption. ${ }^{38}$

The study arms START-B and START-L accrued patients with imatinib-resistant/intolerant CML in myeloid blast crisis ( $n=74$ ) or imatinib-resistant/intolerant CML in lymphoid blast crisis $(n=42)$, respectively. ${ }^{39}$ The starting dasatinib dose was $70 \mathrm{mg}$ bid; patients with a poor initial response were treated with doses up to $100 \mathrm{mg}$ bid, whereas the dose was reduced to $50 \mathrm{mg}$ bid and then to $40 \mathrm{mg}$ bid in patients who experienced toxicity. At 8 months, dasatinib induced major hematologic response rates of $34 \%$ in the START-B trial and $31 \%$ in the START-L trial. Respective rates of major cytogenetic response were $31 \%$ and $50 \%$. Eighty-six percent of major cytogenetic responses were complete cytogenetic responses. At 8 months, the rates of progression-free survival were $88 \%$ in the START-B arm and $46 \%$ in the START-L arm..$^{39}$ In both studies the most likely cause of discontinuation of therapy was disease progression followed by study drug toxicity in the START-B trial and death in the START-L trial. Patients with $B C R-A B L$ mutations E255K, T312I and F486S did not respond to dasatinib. The hematologic responses were durable, and dasatinib was well tolerated except for hematologic toxicity that was reversible with dose interruption or dose reduction. ${ }^{39}$

In the START-C arm 186 patients in chronic phase CML who were resistant to imatinib $>600 \mathrm{mg}$ /day or intolerant to imatinib secondary to adverse events were treated with dasatinib $70 \mathrm{mg}$ po twice daily. ${ }^{40}$ With a median duration of dasatinib therapy of 8 months, the rates of complete hematologic and major cytogenetic response were $90 \%$ and $52 \%$, respectively. Major cytogenetic responses were maintained in $96 \%$ to $100 \%$ of patients. More importantly, dasatinib induced molecular responses and reduced BCR-ABL/ABL transcript ratios from $66 \%$ at baseline to $2.6 \%$ at 9 months. The most common toxicity was myelosuppression. Responses were noted in patients with several $B C R-A B L$ mutations except for the T315I mutation. With a minimum follow-up of 8 months, the progression-free 
survival rate was $92 \%$, and $9 \%$ of patients discontinued therapy because of adverse events. ${ }^{40}$

In the START-R arm in patients with imatinib-resistant, chronic phase CML, patients were randomized 2:1 to receive dasatinib $70 \mathrm{mg}$ po bid $(\mathrm{n}=101)$ or imatinib $400 \mathrm{mg}$ po bid $(\mathrm{n}=49) .{ }^{41}$ Dasatinib therapy induced higher rates of complete hematologic response ( $93 \%$ vs $82 \%$, respectively; $\mathrm{p}=0.034)$, major cytogenetic response $(52 \%$ vs $33 \%$, respectively; $\mathrm{p}=0.023)$, and complete cytogenetic response ( $40 \%$ vs $16 \%$; $p=0.004)$ compared with imatinib. With a median follow-up of 15 months, dasatinib was also associated with lower rates of treatment failure $(p<0.001)$ and higher rates of progression-free survival $(p<0.001)$ compared with imatinib. Dasatinib was also associated with a higher incidence of pleural effusion $(17 \%$ vs $0 \%$ with imatinib), whereas imatinib was associated with higher rates of superficial edema ( $42 \%$ vs $15 \%)$ and fluid retention ( $45 \%$ vs $30 \%) .{ }^{41}$ Cytopenias were more frequent and severe with dasatinib. These results suggested that for patients in the chronic phase of CML who did not respond to the standard imatinib dose, dasatinib was an extremely effective treatment option. ${ }^{41}$

In an interim analysis of another phase II study, dasatinib $140 \mathrm{mg} /$ day po was given to 36 patients with imatinibresistant or imatinib-intolerant $\mathrm{Ph}(+) \mathrm{ALL}$ and CML in lymphoid blast crisis. ${ }^{42}$ With a minimum follow-up of 8 months, dasatinib resulted in a major hematologic response in $42 \%$ of patients, $67 \%$ of whom remained progression free, and resulted in a complete cytogenetic response in $58 \%$ of patients. Overall, $6 \%(2 / 36)$ of patients discontinued therapy as a result of adverse events, with the most common severe adverse event being febrile neutropenia. ${ }^{42}$

\section{Phase III trials}

A phase III trial explored whether dasatinib administered twice daily would be better tolerated compared to daily dosing (Table 1). Overall, 670 imatinib-resistant or -intolerant patients with chronic phase CML were randomized into 4 treatment arms: $100 \mathrm{mg}$ daily, $50 \mathrm{mg}$ twice daily, $140 \mathrm{mg}$ once daily, and $70 \mathrm{mg}$ twice a day. Response rates were comparable among the four arms. The rates of major cytogenetic response ranged from $54 \%$ to $59 \%$ and complete cytogenetic responses ranged from $41 \%$ to $45 \%$ across the four arms. The hematologic response rate ranged from $86 \%$ to $92 \%$. There were no significant differences in progression-free survival, time to response and duration of cytogenetic response among the four arms. The daily $100 \mathrm{mg}$ dose was associated with a lower incidence of toxicities, such as pleural effusion, and thrombocytopenia and with fewer dose interruptions compared with the $70 \mathrm{mg}$ po twice daily dose. ${ }^{43}$

Based on these findings, dasatinib was approved by the FDA at $100 \mathrm{mg}$ po once daily in patients with chronic phase CML and at $70 \mathrm{mg}$ po twice daily in patients with accelerated or blastic phase CML.

\section{Other studies}

Dasatinib has also shown activity in patients with CML who failed nilotinib therapy. In a retrospective analysis of dasatinib in patients with all phases of CML, who failed imatinib and nilotinib therapy, 23 patients were treated with dasatinib $140 \mathrm{mg} /$ day (except for one patient, $240 \mathrm{mg} /$ day). ${ }^{44}$ The response rate to dasatinib was $57 \%$. The complete hematologic response was $43 \%$ (30\% of whom achieved a cytogenetic response, including 2 complete cytogenetic responses). None of the two patients with the T315I mutation responded to dasatinib. After a median follow-up of 34 weeks, $30 \%$ of patients died and $48 \%$ continued on dasatinib therapy. These results provided evidence that resistance to one TKI can be reversed with the use of a different TKI. ${ }^{44}$

\section{Mutational analysis}

Patients resistant to TKIs frequently bear point mutations within the ABL kinase domain of the BCR-ABL fusion protein. The most frequent mutations are located in the ATP-binding loop, namely the P-loop. These mutations are thought to have greater oncogenic activity compared to other mutations and wild-type $B C R-A B L$. Dasatinib has marked activity in patients resistant to imatinib, including those with mutations within the P-loop. Evolving data suggest that dasatinib may be more effective than nilotinib in patients bearing P-loop mutations. ${ }^{45}$

Methods used for mutational analysis include direct sequencing (sensitivity, $20 \%$ to $25 \%$ ); pyrosequencing (sensitivity, $1 \%-5 \%$ ); quantitative mutation-specific RQ-PCR (sensitivity, $0.01 \%-0.1 \%$ ); and liquid bead-array (sensitivity, $5 \%-10 \%$ ). Other methods include denaturing high performance liquid chromatography, targeted microarrays, and liquid bead arrays. ${ }^{46}$ Quantitative mutation detection assays such as PCR-based pyrosequencing and mutation-specific quantitative PCR, as well as digital PCR applications using microfluidic separation, have also been used. The quantitative methods are used to monitor clinical outcomes with the novel TKIs, particularly against the T315I mutation. ${ }^{46}$

Indications for BCR-ABL kinase domain mutational analysis include (a) inadequate initial response to TKIs or evidence of loss of response and (b) advanced phase CML or 
progression to accelerated or blast phase CML. Mutational analysis could be repeated in these patients if they fail to respond to a TKI or if having responded, they subsequently have rising numbers of BCR-ABL transcripts. ${ }^{47}$ Some investigators recommend the use of mutational analysis for a $\geq 10$-fold increase in BCR-ABL transcript levels, although smaller rises in BCR-ABL transcript levels alone may also be predictive of mutation development. However, increasing BCR-ABL transcript levels alone is not a standard criterion because standardization of BCR-ABL RQ-PCR is not optimized. $^{46}$

In a recent report of mutational analysis performed in 1043 patients with CML treated with dasatinib, dasatinib demonstrated broad efficacy, resulting in durable responses in patients with any $B C R-A B L$ mutation except for T315124 (pre-existence or selection of the T315I mutant constitutes the most common mechanism of resistance to dasatinib). ${ }^{48}$ Of 1043 patients, $402(39 \%)$ had a $B C R-A B L$ mutation. The proportion of patients with a $B C R-A B L$ mutation was higher among patients with imatinib resistance ( $48 \%$ of 805 ) compared with imatinib-intolerant patients ( $8 \%$ of 238 ). The investigators reported high response rates and durability of responses in patients with mutations that were highly resistant to imatinib, such as L248, Y253, E255, F359, and H396. The efficacy of dasatinib was also shown in patients with any of these 5 imatinib-resistant mutations with a dasatinib cellular $\mathrm{IC}_{50}>3 \mathrm{nM}$, excluding T315I. More interestingly, patients bearing mutations with a dasatinib $\mathrm{IC}_{50}<3 \mathrm{nM}$ or unknown $\mathrm{IC}_{50}$ had similar response rates and durability of response to those without a $B C R-A B L$ mutation. Responses in patients with a T315I mutation were rare $\left(\mathrm{IC}_{50}>200 \mathrm{nM}\right.$; $\mathrm{n}=21$ ). Overall, 162 patients were followed with mutational analysis: $26 \%$ retained a $B C R-A B L$ mutation, $26 \%$ lost a mutation, $27 \%$ developed a mutation, and the remaining belonged to $>1$ mutational category. ${ }^{24}$

Interestingly, F317L is another emerging mutation that has been observed in dasatinib-resistant patients. ${ }^{48}$ In addition, although dasatinib significantly inhibits CrkL phosphorylation and reduces the total number of $\mathrm{CD} 34^{+} \mathrm{CD} 38^{-} \mathrm{CML}$ cells compared to imatinib, it does not appear to eliminate the most quiescent fraction of stem cells. ${ }^{32,49}$

\section{Toxicity}

Dasatinib is generally well tolerated. The most common non-hematologic adverse events in clinical trials with dasatininb included gastrointestinal symptoms, such as diarrhea, nausea, vomiting, anorexia, abdominal pain and anorexia; headache; peripheral edema; and pleural effusion.
Fewer than $10 \%$ of patients reported mucositis, stomatitis, abdominal distention, constipation, colitis, dysphagia, anal fissure, upper gastrointestinal ulcers, pancreatitis and esophagitis. Pleural and pericardial effusion, severe pulmonary edema, pulmonary hypertension, and ascites were less common. Ascites and pleural effusion are usually reversible with temporary dose interruption, diuretics and steroids.

Myelosuppression is common and it is reversible by interruption, reduction or discontinuation of the myelosuppression-inducing dasatinib dose. Grade 3-4 myelosuppression is more frequent in patients with accelerated or blastic phase CML and constitutes the most common reason for drug interruption. Thrombocytopenia is more significant than neutropenia and its associated rates of gastrointestinal bleeding and central nervous system hemorrhage are $<4 \%$ and $<1 \%$, respectively, whereas the rate of other severe hemorrhages is approximately $2 \%$. Dasatinib-induced thrombocytopenia associated with hemorrhagic complications suggests that patients receiving anticoagulation or antiplatelet agents should be treated with caution.

Cardiac adverse events are infrequent and include left ventricular dysfunction, cardiac failure, cardiomyopathy, ventricular failure, diastolic dysfunction, and QT-interval prolongation. Skin sequelae include erythema, erythema multiforme, pustular rash, pruritic rash, skin exfoliation, skin irritation, systemic lupus erythematous, vesicular rash, and maculopapular rash. Fewer than $10 \%$ of patients develop acne, alopecia, skin ulcer, pigmentation disorder, panniculitis, photosensitivity, nail disorders, and palmar plantar erythrodysesthesia.

The use of dasatinib is also associated with hypocalcemia, hypophosphatemia, transaminitis and creatinine elevation. Symptoms from the central nervous system are noted in $<10 \%$ of patients and include neuropathy, dizziness, dysgeusia, somnolence, tremor, syncope, transient ischemic attack, cerebrovascular disease and seizures. Other toxicities include hypersensitivity, gynecomastia, irregular menses, vertigo, appetite disturbances, increased triglycerides, cholesterol, pyrexia and fatigue.

\section{Hematopoietic stem cell transplantation}

The use of TKIs has limited the use of this previously commonly used therapeutic strategy in CML. According to the 2009 NCCN recommendations, hematopoietic stem cell transplantation should be offered as an option to patients who (a) have no cytogenetic response at 6 months, (b) have cytogenetic relapse at 12 and 18 months following initial hematologic remission, (c) have partial cytogenetic response 
at 18 months and (d) have T315I mutation and failed to respond to imatinib, dasatinib or nilotinib.

\section{Future studies}

Dasatinib has been shown to synergize with novel TKIs in producing growth inhibition and apoptosis in CML cells. ${ }^{50}$ Therefore, carefully designed clinical trials with combined TKIs may improve the prognosis of imatinib-resistant CML. For instance, patients with imatinib-resistant CML and 2 subclones, one carrying F359V and the other F317L, could benefit from a combination therapy of dasatinib plus nilotinib, if hematopoietic stem cell transplantation is not an option.

\section{Conclusion}

Dasatinib is an effective therapy for patients with CML. It is more effective than high-dose imatinib in patients who failed standard dose imatinib. Because dasatinib enters the central nervous system, patients whose disease relapses in the central nervous system may benefit from dasatinib. However, new agents are needed for patients with a T3151 mutation, since patients with this mutation are resistant to dasatinib. Other second-generation TKIs with activity in CML include nilotinib, bosutinib and INNO 406. New molecules, such as the inhibitor of Aurora family serine-threonine kinases, MK0457, which has antileukemic activity in CML associated with the T315I mutation, are being investigated in CML.

\section{Disclosures}

The authors disclose no conflicts of interest.

\section{References}

1. Goldman JM, Melo JV. Chronic myeloid leukemia-advances in biology and new approaches to treatment. $N$ Engl J Med. 2003;349:1451-1464.

2. Kurzrock R, Gutterman JU, Talpaz M. The molecular genetics of Philadelphia chromosome-positive leukemias. NEngl J Med. 1988;319: 990-998.

3. Calabretta B, Perrotti D. The biology of CML blast crisis. Blood. 2004; 103:4010-4022.

4. Druker BJ, Tamura S, Buchdunger E, et al. Effects of a selective inhibitor of the Abl tyrosine kinase on the growth of Bcr-Abl positive cells. Nat Med. 1996;2:561-566.

5. Sawyers CL. Chronic myeloid leukemia. N Engl J Med. 1999;340: $1330-1340$.

6. Cohen MH, Williams G, Johnson JR, et al. Approval summary for imatinib mesylate capsules in the treatment of chronic myelogenous leukemia. Clin Cancer Res. 2002;8:935-942.

7. Gambacorti-Passerini C, le Coutre P, Mologni L, et al. Inhibition of the ABL kinase activity blocks the proliferation of BCR/ABL+ leukemic cells and induces apoptosis. Blood Cells Mol Dis. 1997;23: 380-394.

8. Druker J, O'Brien S, Larson RA. Long-term benefits of imatinib (IM) for patients newly diagnosed with chronic myelogenous leukemia in chronic phase (CML-CP): The 5-year update from the IRIS study. J Clin Oncol. (Meeting Abstracts) 2006;24(18S P1): Abstract 6506.
9. Hochhaus A, Druker BJ, Larson R, O’Brien SG, Gathmann I, Guilhot F. IRIS 6-year follow-up: Sustained survival and declining annual rate of transformation in patients with newly diagnosed chronic myeloid leukemia in chronic phase (CML-CP) treated with imatinib (abstract 25). Blood. 2007;110. p. 16a.

10. O'Brien SG, Guilhot F, Larson RA, et al. Imatinib compared with interferon and low-dose cytarabine for newly diagnosed chronic-phase chronic myeloid leukemia. N Engl J Med. 2003;348:994-1004.

11. Baccarani M, Saglio G, Goldman J, et al. Evolving concepts in the management of chronic myeloid leukemia: recommendations from an expert panel on behalf of the European LeukemiaNet. Blood. 2006;108:1809-1820.

12. Shah NP, Sawyers CL. Mechanisms of resistance to STI571 in Philadelphia chromosome-associated leukemias. Oncogene. 2003;22:7389-7395.

13. Gorre ME, Mohammed M, Ellwood K, et al. Clinical resistance to STI-571 cancer therapy caused by BCR-ABL gene mutation or amplification. Science. 2001;293:876-880.

14. Soverini S, Martinelli G, Rosti G, et al. ABL mutations in late chronic phase chronic myeloid leukemia patients with up-front cytogenetic resistance to imatinib are associated with a greater likelihood of progression to blast crisis and shorter survival: a study by the GIMEMA Working Party on Chronic Myeloid Leukemia. J Clin Oncol. 2005;23:4100-4109.

15. Cowan-Jacob SW, Guez V, Fendrich G, et al. Imatinib (STI571) resistance in chronic myelogenous leukemia: molecular basis of the underlying mechanisms and potential strategies for treatment. Mini Rev Med Chem. 2004;4:285-299.

16. Hochhaus A, Kreil S, Corbin AS, et al. Molecular and chromosomal mechanisms of resistance to imatinib (STI571) therapy. Leukemia. 2002;16:2190-2196.

17. Illmer T, Schaich M, Platzbecker U, et al. P-glycoprotein-mediated drug efflux is a resistance mechanism of chronic myelogenous leukemia cells to treatment with imatinib mesylate. Leukemia. 2004;18:401-408.

18. Thomas J, Wang L, Clark RE, Pirmohamed M. Active transport of imatinib into and out of cells: implications for drug resistance. Blood. 2004;104:3739-3745.

19. Gambacorti-Passerini C, Barni R, le Coutre P, et al. Role of alpha1 acid glycoprotein in the in vivo resistance of human BCR-ABL(+) leukemic cells to the abl inhibitor STI571. J Natl Cancer Inst. 2000;92:1641-1650.

20. Burger H, van Tol H, Boersma AW, et al. Imatinib mesylate (STI571) is a substrate for the breast cancer resistance protein (BCRP)/ABCG2 drug pump. Blood. 2004;104:2940-2942.

21. Rix U, Hantschel O, Durnberger G, et al. Chemical proteomic profiles of the BCR-ABL inhibitors imatinib, nilotinib, and dasatinib reveal novel kinase and nonkinase targets. Blood. 2007;110: 4055-4063.

22. Shah NP, Tran C, Lee FY, Chen P, Norris D, Sawyers CL. Overriding imatinib resistance with a novel ABL kinase inhibitor. Science. 2004;305:399-401.

23. O'Hare T, Walters DK, Stoffregen EP, et al. In vitro activity of Bcr-Abl inhibitors AMN107 and BMS-354825 against clinically relevant imatinib-resistant Abl kinase domain mutants. Cancer Res. 2005;65:4500-4505.

24. Muller MC, Cortes J, Kim D-W, et al. Dasatinib efficacy in patients with chronic myeloid leukemia in chronic phase and preexisting BCR-ABL mutations (abstract 449). Blood. 2008;112(11):171.

25. Redaelli S, Piazza R, Rostagno R, et al. Activity of bosutinib, dasatinib, and nilotinib against 18 imatinib-resistant BCR/ABL mutants. J Clin Oncol. 2009;27:469-471.

26. Apperley JF. Part I: mechanisms of resistance to imatinib in chronic myeloid leukaemia. Lancet Oncol. 2007;8:1018-1029.

27. Tokarski JS, Newitt JA, Chang CY, et al. The structure of Dasatinib (BMS-354825) bound to activated ABL kinase domain elucidates its inhibitory activity against imatinib-resistant ABL mutants. Cancer Res. 2006;66:5790-5797. 
28. Brave M, Goodman V, Kaminskas E, et al. Sprycel for chronic myeloid leukemia and Philadelphia chromosome-positive acute lymphoblastic leukemia resistant to or intolerant of imatinib mesylate. Clin Cancer Res. 2008;14:352-359.

29. Lombardo LJ, Lee FY, Chen P, et al. Discovery of N-(2-chloro6-methyl- phenyl)-2-(6-(4-(2-hydroxyethyl)- piperazin-1-yl)-2methylpyrimidin-4-ylamino)thiazole-5-carboxamide (BMS-354825), a dual Src/Abl kinase inhibitor with potent antitumor activity in preclinical assays. J Med Chem. 2004;47:6658-6661.

30. Konig H, Copland M, Chu S, Jove R, Holyoake TL, Bhatia R. Effects of dasatinib on Src kinase activity and downstream intracellular signaling in primitive chronic myelogenous leukemia hematopoietic cells. Cancer Res. 2008;68:9624-9633.

31. Shah NP, Kasap C, Weier C, et al. Transient potent BCR-ABL inhibition is sufficient to commit chronic myeloid leukemia cells irreversibly to apoptosis. Cancer Cell. 2008;14:485-493.

32. Copland M, Hamilton A, Elrick LJ, et al. Dasatinib (BMS-354825) targets an earlier progenitor population than imatinib in primary CML but does not eliminate the quiescent fraction. Blood. 2006;107: 4532-4539.

33. Kim DH, Kamel-Reid S, Chang H, et al. Natural killer or natural killer/T cell lineage large granular lymphocytosis associated with dasatinib therapy for Philadelphia chromosome positive leukemia Haematologica. 2009;94:135-139.

34. Porkka K, Koskenvesa P, Lundan T, et al. Dasatinib crosses the blood-brain barrier and is an efficient therapy for central nervous system Philadelphia chromosome-positive leukemia. Blood. 2008;112: 1005-1012.

35. Kamath AV, Wang J, Lee FY, Marathe PH. Preclinical pharmacokinetics and in vitro metabolism of dasatinib (BMS-354825): a potent oral multi-targeted kinase inhibitor against Src and BCR-ABL. Cancer Chemother Pharmacol. 2008;61:365-376.

36. Brendel C, Scharenberg C, Dohse M, et al. Imatinib mesylate and nilotinib (AMN107) exhibit high-affinity interaction with ABCG2 on primitive hematopoietic stem cells. Leukemia. 2007;21:1267-1275.

37. Talpaz M, Shah NP, Kantarjian H, et al. Dasatinib in imatinibresistant Philadelphia chromosome-positive leukemias. $N$ Engl J Med. 2006;354:2531-2541.

38. Guilhot F, Apperley J, Kim DW, et al. Dasatinib induces significant hematologic and cytogenetic responses in patients with imatinib-resistant or -intolerant chronic myeloid leukemia in accelerated phase. Blood. 2007;109:4143-4150.

39. Cortes J, Rousselot P, Kim DW, et al. Dasatinib induces complete hematologic and cytogenetic responses in patients with imatinib-resistant or -intolerant chronic myeloid leukemia in blast crisis. Blood. 2007;109:3207-3213.

40. Hochhaus A, Kantarjian HM, Baccarani M, et al. Dasatinib induces notable hematologic and cytogenetic responses in chronic-phase chronic myeloid leukemia after failure of imatinib therapy. Blood. 2007;109:2303-2309.
41. Kantarjian H, Pasquini R, Hamerschlak N, et al. Dasatinib or high-dose imatinib for chronic-phase chronic myeloid leukemia after failure of first-line imatinib: a randomized phase 2 trial. Blood. 2007;109:5143-5150.

42. Ottmann O, Dombret H, Martinelli G, et al. Dasatinib induces rapid hematologic and cytogenetic responses in adult patients with Philadelphia chromosome positive acute lymphoblastic leukemia with resistance or intolerance to imatinib: interim results of a phase 2 study. Blood. 2007;110:2309-2315.

43. Shah NP, Kantarjian HM, Kim DW, et al. Intermittent target inhibition with dasatinib $100 \mathrm{mg}$ once daily preserves efficacy and improves tolerability in imatinib-resistant and -intolerant chronic-phase chronic myeloid leukemia. J Clin Oncol. 2008;26:3204-3212.

44. Quintas-Cardama A, Kantarjian H, Jones D, et al. Dasatinib (BMS-354825) is active in Philadelphia chromosome-positive chronic myelogenous leukemia after imatinib and nilotinib (AMN107) therapy failure. Blood. 2007;109:497-499.

45. Cang S, Liu D. P-loop mutations and novel therapeutic approaches for imatinib failures in chronic myeloid leukemia. $J$ Hematol Oncol. 2008; $1: 15$

46. Jones D, Kamel-Reid S, Bahler D, et al. Laboratory practice guidelines for detecting and reporting BCR-ABL drug resistance mutations in chronic myelogenous leukemia and acute lymphoblastic leukemia: a report of the Association for Molecular Pathology. J Mol Diagn. 2009;11:4-11.

47. Hughes T, Deininger M, Hochhaus A, et al. Monitoring CML patients responding to treatment with tyrosine kinase inhibitors: review and recommendations for harmonizing current methodology for detecting BCR-ABL transcripts and kinase domain mutations and for expressing results. Blood. 2006;108:28-37.

48. Soverini S, Martinelli G, Colarossi S, et al. Presence or the emergence of a F317L BCR-ABL mutation may be associated with resistance to dasatinib in Philadelphia chromosome-positive leukemia. J Clin Oncol. 2006;24:e51-e2.

49. Melo J, Chuah C. Novel agents in CML therapy: tyrosine kinase inhibitors and beyond. Am Soc Hematol. 2008:427-435.

50. Copland M, Pellicano F, Richmond L, et al. BMS-214662 potently induces apoptosis of chronic myeloid leukemia stem and progenitor cells and synergizes with tyrosine kinase inhibitors. Blood 2008;111:2843-2853

51. Hochhaus A, Baccarani M, Deininger M, et al. Dasatinib induces durable cytogenetic responses in patients with chronic myelogenous leukemia in chronic phase with resistance or intolerance to imatinib. Leukemia. 2008;22:1200-1206.

52. Cortes J, Kim DW, Raffoux E, et al. Efficacy and safety of dasatinib in imatinib-resistant or -intolerant patients with chronic myeloid leukemia in blast phase. Leukemia. 2008;22:2176-2183. 
